\title{
Efficient mentorship
}

\section{Sathiadas MG}

Mentoring is the process by which an experienced person provides guidance, support, and encouragement to a less experienced person. Such relationships are based on consideration, camaraderie, commonality, and confidentiality (1).

Mentoring has different models and it changes with time. What ever the model the mentor and mentee can work out details, setting learning goals and avoid jeopardizing the goal of a mentorship programme.

The apprenticeship model has a heirachial approach and the trainee is mentored and taught by a more experienced professional. The cloning model is to mentor in plan of succession and the mentee is groomed into the role. The nurturing model creates a safe and open environment in which mentee discusses personal issues and learn by themselves with the mentor acting as a facilitator. The friendship model is the mentor and mentee maintain the same professional level rather than the hierarchical approach (2).

Many institutions are addressing this by formalizing the process and assigning mentors for medical students and junior doctors, although it is up to the mentees to find someone they respect and trust to help them reach their objectives. Once the partnership is underway, both partners must work to sustain the relationship and help it flourish (3). Mentoring programmes have been implemented as a specific career-advancement tool in the training and further education of various groups in the medical profession (4). A review of the medline literature on mentoring programmes indicated that majority of the programmes lack a concrete structure as well as a short- and long-term evaluation. Main goals of these publications are to increase professional competence in research and in further specialization to build up a professional network for the mentees; no statements are to be found on the advantages for the mentors. Due to this most programmes fail to achieve the appropriate effectiveness and achievement (4).

Challenges of effective mentorship is mainly due to the workload experienced by the mentors.
The endless string of demanding tasks at work on deadlines, meetings, projects, and ongoing training modules all limiting our time to refuel. As an energy-saving measure, we may cut corners. One task that commonly falls down on the priority list is mentoring. In the face of a pandemic with no end in sight, we must preserve our fuel supplies while we mentor others.

To start the mentoring process the mentors and mentees must draft a document of standards for future use. Mentees must create the agenda, organize calendar invitations, and complete action items. A templated list at the start of a relationship can offer friendly but direct guidelines to each mentee. During your relationship, you can revisit the document together and optimize further. Provide context, informing the mentee that these standards will provide organization and leadership skills and be focused on their needs (5).

An important part in mentoring is the time you can spend together. A single mentee might need two to six hours per year, depending on the mentee's needs. Share your time budget with the mentee. This allows mentees to see the mentor's time as a currency and develop thoughts and questions prior to reaching out.

Group mentoring holds great potential for undergraduate medical education. However, the scientific literature on this is sparse. Group mentorship programs benefit from being longitudinal and mandatory. Ideally, they should provide opportunities throughout undergraduate medical education for regular meetings where discussions and personal reflection occur in a supportive environment (5). This method is a feasible option especially in resource limited settings. This has advantages in regards to timing and scheduling meetings as well as other advantages, too: In the group setting, mentees can share perspective with each other and offer peer-to-peer mentorship. This establishes support and validation when mentees hear similar struggles and unmet aspirations. It also allows the mentees to build their trusted spaces and personal networks as a group by discussing what would otherwise be private matters in a 1:1 meeting. 
Group mentoring sessions do not have to be inperson as we have learned in the Covid-19 era, they work well virtually. Using the virtual platform to mentor will overcome the challenges of time and space needed for efficient mentoring. Additionally, consider using group messaging apps to help in mentoring. This keeps the mentor present and engaged while allowing flexibility to disconnect and hold notifications.

Mentoring should not burn and drain energy. It can be fuel-efficient and effortless. Look for ways to communicate expectations, schedule effectively, and consolidate mentees. These strategies provide room to go farther on the journey as a mentor.

\section{References:}

1. Ratnapalan S. (2010). Mentoring in medicine. Canadian family physician Medecin de famille canadien, 56(2), 198.
2. Straus SE, Chatur F, Taylor M. Issues in the men tor-mentee relationship in academic medicine: a qualitative study. Acad Med. 2009;84(1):1359

3. Rose GL, Rukstalis MR, Schuckit MA. Infor mal mentoring between faculty and medical students. Acad Med. 2005;80(4):344-8

4. Buddeberg-Fischer B, Herta KD. Formal mentoring programmes for medical students and doctors--a review of the Medline literature. Med Teach. 2006 May;28(3):24857. doi: 10.1080/01421590500313043. PMID: 16753724.

5. Skjevik EP, Boudreau JD, Ringberg U, Schei E, Stenfors T, Kvernenes M, Ofstad EH. Group mentorship for undergraduate medical students-a systematic review. Perspect Med Educ. 2020 Oct;9(5):272-280. doi: 10.1007/ S40037-020-00610-3. PMID: 32820416; PMCID: PMC7550430. 